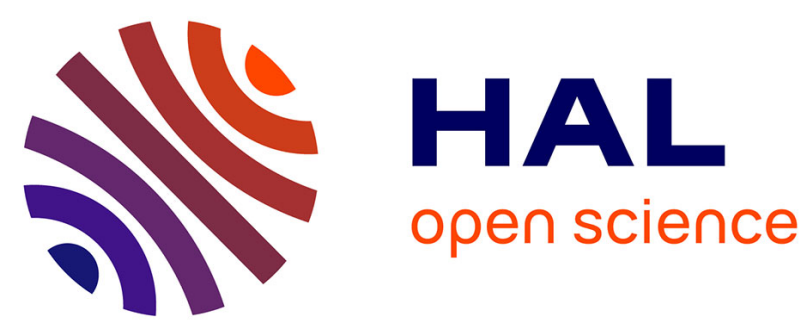

\title{
Assisted test generation strategy for non-intrusive machine learning indirect test of millimeter-wave circuits
}

F. Cilici, Manuel J. Barragan, Salvador Mir, Estelle Lauga-Larroze, Sylvain Bourdel

\section{- To cite this version:}

F. Cilici, Manuel J. Barragan, Salvador Mir, Estelle Lauga-Larroze, Sylvain Bourdel. Assisted test generation strategy for non-intrusive machine learning indirect test of millimeter-wave circuits. 23rd IEEE European Test Symposium (ETS'2018), May 2018, Bremen, Germany. pp.1-6, 10.1109/ETS.2018.8400689 . hal-01922313

\section{HAL Id: hal-01922313 \\ https://hal.science/hal-01922313}

Submitted on 2 Oct 2020

HAL is a multi-disciplinary open access archive for the deposit and dissemination of scientific research documents, whether they are published or not. The documents may come from teaching and research institutions in France or abroad, or from public or private research centers.
L'archive ouverte pluridisciplinaire HAL, est destinée au dépôt et à la diffusion de documents scientifiques de niveau recherche, publiés ou non, émanant des établissements d'enseignement et de recherche français ou étrangers, des laboratoires publics ou privés.

\section{(ㄷ)(1) $\$$}

Distributed under a Creative Commons Attribution - NonCommercial| 4.0 International 


\title{
Assisted test design for non-intrusive machine learning indirect test of millimeter-wave circuits
}

\author{
Florent Cilici*†, Manuel J. Barragan*, Salvador Mir*, Estelle Lauga-Larroze ${ }^{\dagger}$ and Sylvain Bourdel ${ }^{\dagger}$ \\ ${ }^{*}$ Univ. Grenoble Alpes, CNRS, Grenoble INP ${ }^{1}$, TIMA F-38000 Grenoble, France \\ ${ }^{\dagger}$ Université Grenoble-Alpes, IMEP-LAHC Minatec 38016 Grenoble, France
}

\begin{abstract}
The functional test of millimeter-wave (mm-wave) circuitry in the production line is a challenging task that requires costly dedicated test equipment and long test times. Machine learning indirect test offers an appealing alternative to standard mm-wave functional test by replacing the direct measurement of the circuit performances by a set of indirect measurements, usually called signatures. Machine learning regression algorithms are then used to map signatures and performances. In this work, we present a generic and automated methodology for finding an appropriate set of indirect measurements and assisting the designer with the necessary Design-for-Test circuit modifications. In order to avoid complex design modifications of $\mathbf{m m}$-wave circuitry, the proposed strategy is targeted at generating a set of non-intrusive indirect measurements using process variation sensors not connected to the Device Under Test (DUT). The proposed methodology is demonstrated on a $60 \mathrm{GHz}$ Power Amplifier designed in STMicroelectronics $55 \mathrm{~nm}$ BiCMOS technology.
\end{abstract}

\section{INTRODUCTION}

Exploiting the millimeter-wave frequency band has been proposed as an answer to the ever-increasing need of transmitting large volumes of data. Nowadays, mm-wave devices are present in high-end communication systems (e.g., military communication systems) and are expanding into consumer electronics (e.g., car radars for autonomous cars, future communication standards such as 5G, etc.) [1]-[3]. However, testing mm-wave circuitry in the production line is still based on complex functional measurements at very high frequencies that demand costly dedicated test equipment and represent a bottleneck in the production.

Machine learning indirect test, first introduced as the socalled alternate test strategy in [4], is a possible solution to alleviate the complexity and cost associated to testing mmwave circuitry. The aim of machine learning indirect test is to replace the measurement of complex functional specifications by a set of simpler signatures. A machine learning regression algorithm is used to map the signatures to the specifications. The usual approach to machine learning indirect test is based on supervised machine learning algorithms. The process is developed in two stages: a learning stage, and a testing stage. During the learning stage both performance parameters and signatures are measured from a set of training devices. A machine learning algorithm is then trained over the two sets of measurements to build a mapping model. In the testing stage, signatures are measured for each DUT, and performances are inferred by using the mapping model obtained in the previous

${ }^{1}$ Institute of Engineering Univ. Grenoble Alpes stage. This indirect test framework has the benefit of a simple interpretation of the test outcome since the estimated specifications are directly compared to their test acceptance windows in a conventional manner. Moreover, indirect measurements are devised to be cheaper than the direct measurement of functional specifications.

Furthermore, in the last few years an indirect test strategy based on non-intrusive sensors has been proposed [5]-[7]. This non-intrusive approach relies on process-aware sensors that are not electrically connected to the DUT. Signatures are extracted from these sensors without modifying the topology of the DUT or perturbing its functionality. This approach is particularly interesting for mm-wave circuits, since loading internal nodes to extract a measurement requires a high design effort and may have a significant impact on the DUT performance.

However, machine learning indirect tests are not free of shortcomings. One of the key points that in our opinion limits the adoption of the technique is the lack of a reliable and automated tool for proposing appropriate signatures that are strongly correlated to the specifications. Previous efforts in this line include the optimization strategy in [8], where a piece-wise linear stimulus is optimized to minimize the prediction error of the regression model. While this is a sound strategy, the choice and the parametrization of the input stimulus are still ad hoc. Usually, the initial set of signatures is proposed based on expert design knowledge and may contain unreliable information, noisy signatures, redundant data, or even signatures that are not correlated to the target performances. Moreover, there is not a systematic approach for generating an appropriate set of signatures for non-intrusive indirect tests, and the process has to be repeated for every different DUT. From the point of view of Electronic Design Automation (EDA) tools, this is similar to developing an Automatic Test Pattern Generator (ATPG) for a given test campaign. Such a systematic approach would provide the set of input measurements (i.e., signatures) that maximizes the quality of the machine learning regression model.

In this work, we propose a generic strategy for finding appropriate indirect measurements for the prediction of a given set of performances in a machine learning non-intrusive indirect test scenario. Moreover, the proposed strategy also assists in the design of the specifically tailored non-intrusive process variation sensors needed for the implementation of the resulting indirect test program. As a key difference to previously presented approaches for feature selection [9]-[12] 
and non-intrusive machine learning indirect tests, the proposed strategy is targeted at test automation and it does not need a priori design knowledge of the DUT, nor an initial set of signatures based on expert knowledge.

This paper is structured as follows. Section II reviews previous work on feature selection techniques and RF/mmwave indirect test, with a special focus on non-intrusive approaches. Section III presents our strategy for automated signature proposal and assisted non-intrusive sensor synthesis. In Section IV we describe the application of the proposed technique to the machine learning indirect test of a $60 \mathrm{GHz}$ PA in a $55 \mathrm{~nm}$ BiCMOS technology. The feasibility of the proposed technique is verified by electrical Monte Carlo (MC) simulations. Finally, Section V summarizes the main contributions of this paper.

\section{PREVIOUS WORK}

Finding the appropriate set of input signatures to build a reliable regression model is a classical problem in the field of Statistics and a variety of feature selection strategies have been proposed to deal with this issue. In this line, different feature selection techniques have been adapted to machine learning indirect test approaches to select the best subset of features within an initial set of potential features [9], [12]-[15].

A direct approach to feature selection consists in preselecting a subset of features based on some statistical observations, before training any regression models. This approach, widely used when the number of initial features is high, is known as filtering. Some filtering techniques recently proposed include Principal Component Analysis (PCA) [13], multivariate distance correlation [14], Kendall's tau correlation metric [12], etc. However, these filtering techniques only capture the most significant variation components. In order to further improve the selection algorithm, it is necessary to resort to wrapper approaches. Basically, a wrapper algorithm uses the machine learning prediction model as a black box within an optimization loop, with the objective of finding the subset of signatures of minimum cost that minimizes the prediction error in an independent validation set [9], [10]. Finally, hybrid filter-wrapper methodologies have been proposed to reduce the computational burden of the wrapper approach. Hybrid techniques guide the search in the input signature space based on the dynamic evaluation of a correlation metric [15].

The main limitation of these feature selection techniques for a fully automated indirect test generation is the lack of a methodology for proposing the initial set of signatures. In this line, some work has been presented for guiding the design of new features based on the missing information in the initial set of input signatures [9].

In this work, we aim at fully automating the proposal of the initial set of signatures for the particular case of nonintrusive machine learning indirect test. Non-intrusive indirect test was first proposed in [5] for mixed-signal test applications, and later extended to the RF and mm-wave domain in [7], [16]. These non-intrusive techniques propose to use process variation sensors that mimic sensitive portions of the
DUT. Then, DC measurements on these sensors, that are not electrically connected to the DUT, are used as test signatures for training machine learning regression models. As it was mentioned above, the technique has obvious advantages for RF and mm-wave test, since the topology of the circuit is not modified and no additional loads are added to the DUT nodes. However, this technique is highly dependent on design knowledge: sensors are proposed based on an expert analysis on the circuit topology and no systematic approach for defining the set of initial signatures is proposed.

\section{PROPOSED APPROACH}

Let us consider a generic non-intrusive machine learning indirect test scenario in which we intend to regress a set of performances of a given DUT from a set of signatures measured from a set of non-intrusive process variation sensors. The problem we address in this work is, given the netlist of a particular DUT in a given technology and a set of target performances, how to systematize the proposal of the set of signatures and the design of the associated sensors.

The methodology that we propose is divided into two interrelated steps. The first step is aimed at unveiling the root causes of parametric performance variation in the simulation environment. The second step is devised to find process variation sensors that are sensitive to the identified set of root causes. Let us describe these steps separately.

\section{A. Step 1: Unveiling the root causes of parametric perfor- mance variation}

The key idea for finding the root causes of performance variation at the design stage is to take advantage of the available information in the MC models included in the Process Design Kit (PDK). Indeed, in a MC simulation environment, we have direct access to the complete set of root causes behind the DUT performance variation, that is, the set of statistical model parameters generated for each MC iteration. There are two sets of statistical parameters, describing global parametric process variations and local random mismatch. The non-intrusive machine learning indirect test approach works under the assumption that the effect of mismatch can be neglected with respect to process variations. Thus, our methodology explores the space of MC global process variation parameters to find the minimum subset that explains the observed parametric performance variation within a given accuracy threshold.

Exploring the space of process variation parameters can be seen as a feature selection problem in which we consider the $\mathrm{MC}$ parameters as candidate features to regress a given target performance using a machine learning regression model. Thus, we propose to adapt the Brownian distance correlation-directed search, previously presented in [15], according to Algorithm 1.

Given a target performance, the proposed algorithm first ranks the MC process parameters with respect to their distance correlation to the target performance and trains an initial regression model with the most correlated parameter. Then, we compute the residues of the fit (i.e. the individual errors 


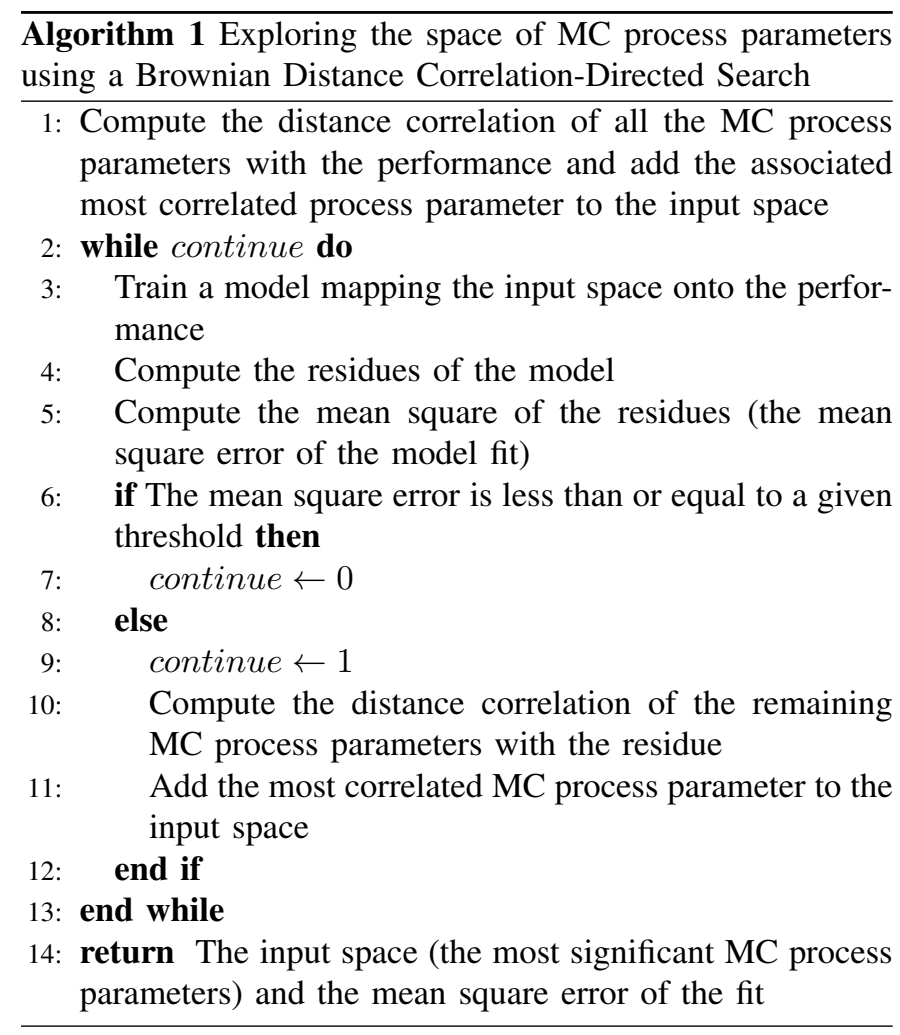

for all the training samples). After that, the algorithm ranks the remaining $\mathrm{MC}$ process parameters with respect to their distance correlation to the residues of the fit and selects the best candidate to be added to the set of the most significant MC parameters. By iterating this procedure, the search is guided so that each iteration of the algorithm should identify a MC parameter that adds relevant information that was previously missing.

A key point of the algorithm above is the concept of correlation, that relies on the evaluation of the Brownian Distance correlation [14], [17]. This non-linear correlation metric is sensitive to multivariate non-linear dependencies. The distance correlation is actually computed between the augmented input space -that is, the matrix of previously selected MC process parameters plus the new candidate- and the vector of residues. The output of the algorithm is the set of $\mathrm{MC}$ process parameters that best explains the variation of the selected target performance. The process can then be iterated to cover the rest of target performances of the considered DUT.

\section{B. Step 2: Assisted design of non-intrusive process variation sensors}

The following step is to devise simple process variation sensors that provide a signature sensitive to the set of the most significant MC process parameters selected by the previous algorithm. Again, we take advantage of the information contained in the PDK of the technology to identify the underlying physical meaning of the selected process parameters. This way we can guide the design of appropriate non-intrusive sensors to target the desired information. Ideally, a dedicated sensor may be designed for each one of the selected process parameters. However, signatures, in practice, may contain contributions from several process parameters. The devised sensors, and their associated signatures, are then stored in a library for further reuse. Indeed, as the methodology is applied to different DUTs in the same technology, sensors may be reused if the same MC process parameters are deemed significant for new DUTs. In fact, once this library covers the complete set of MC parameters, the design of the test protocol becomes a simple test selection problem within the tests in the library. The methodology only calls for human intervention if there is no sensor in the library associated to a given process parameter. This simple strategy leads the proposed methodology to capture the expert knowledge of the designer for the generation of new tests and sensors. It is worth noticing that, since the methodology guides the design of the sensors towards a target $\mathrm{MC}$ process parameter, sensors are actually independent of the topology of the DUT. In fact, no expert design knowledge on the DUT is required for any of the stages of the proposed methodology.

As a sanity check, we implement an automated validation for each developed sensor consisting in applying a Brownian distance correlation directed-search in the complete space of $\mathrm{MC}$ process parameters to train a regression model toward the sensor output signature. The goal of this validation is to ensure that the proposed signature is actually sensitive to the desired MC parameters.

The final output of the proposed test design algorithm for indirect test is the set of non-intrusive sensors and the actual set of measurements (i.e., the signatures associated to the sensors) for the regression of the DUT performances in a machine learning indirect test scenario.

\section{CASe Study}

The selected DUT for our case study is a class A one-stage $\mathrm{mm}$-wave Power Amplifier designed in STMicroelectronics $55 \mathrm{~nm}$ BiCMOS technology. Despite the apparent simplicity of the circuit, it is difficult to identify a priori the dominant source of performance degradation of the PA, since it is affected by variations of both the active and passive components. This makes it an interesting case study for the proposed test generation methodology. Figure 1 shows the transistor-level schematic of the circuit. The PA input and output nodes are matched to a $50 \Omega$ impedance at $60 \mathrm{GHz}$ using microstrip line stubs. The main performance specifications of the designed PA, obtained after post-layout simulation, under typical corner operation conditions are listed in Table I.

The proposed test design methodology has been applied to the selected DUT as a practical case study to illustrate the technique. The goal of this case study is to generate a set of appropriate non-intrusive sensors that provide meaningful signatures for testing the DUT using a machine learning indirect test strategy.

A set of 2000 instances of the selected PA has been generated using the MC models provided in the PDK of the technology. A total of 300 instances of the PA are set 


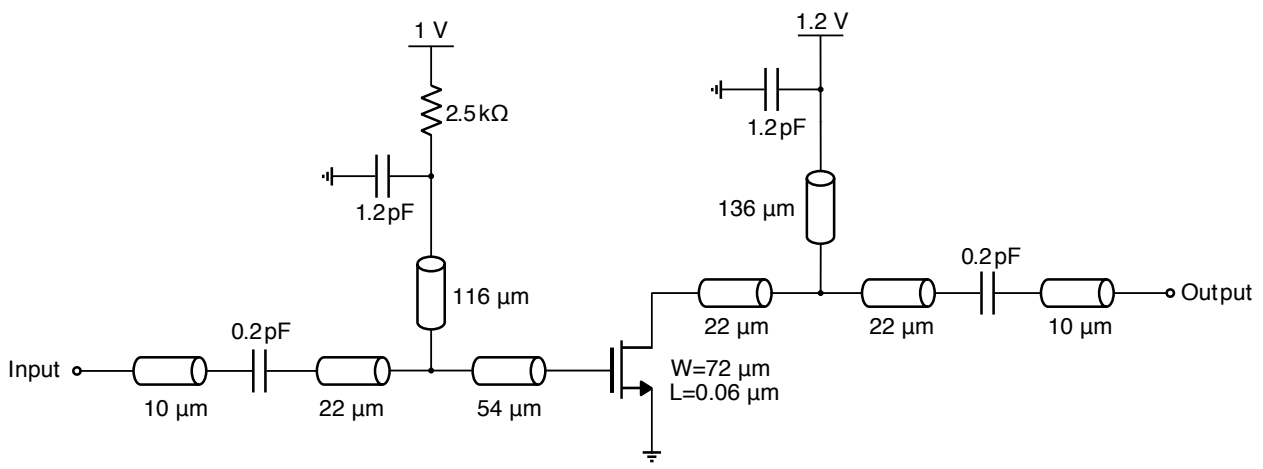

Fig. 1. Transistor level schematic of the Power Amplifier under test

TABLE I

PERFormanCE AND DESIGN PARAMETERS OF THE PA UNDER TEST

\begin{tabular}{lc}
\hline Operating frequency range & 59 to $61 \mathrm{GHz}$ \\
DC current, $I_{D C}$ & $28.2 \mathrm{~mA}$ \\
Power supply, $V_{D D}$ & $1.2 \mathrm{~V}$ \\
Gain $\left(S_{21}\right)$ & $5.6 \mathrm{~dB} @ 60 \mathrm{GHz}$ \\
Power Added Efficiency, $P A E$ & $11 \%$ \\
$S_{11}$ & $<-20 \mathrm{~dB}$ \\
$S_{22}$ & $<-20 \mathrm{~dB}$ \\
$S_{12}$ & $<-13 \mathrm{~dB}$ \\
Input referred 1dB-Compression Point, $C P_{1 d B}$ & $0.7 \mathrm{dBm}$ \\
Saturation output power, $P_{\text {sat }}$ & $10.5 \mathrm{dBm}$ \\
Input referred third-order intercept point, $I I P 3$ & $11 \mathrm{dBm}$ \\
\hline
\end{tabular}

apart to be used as an independent verification set and the remaining instances are used for training. The first step of the proposed methodology, as described above, is to find the rootcauses of parametric variation within the set of MC parameters defined in the technology. The MC simulation of the PA in the selected $55 \mathrm{~nm}$ BiCMOS technology includes 154 independent process parameters $\left\{M C_{1}, \ldots, M C_{154}\right\}$ that define the search space. The exploration of the MC parameter space uses the proposed Brownian distance correlation-directed search for regressing the PA specifications. A perceptron Neural Network is used as machine learning regression algorithm. Due to space limitations, the complete procedure is illustrated for only one of the specifications, i.e. the small signal gain of the PA at $60 \mathrm{GHz}$. The process has to be iterated for the rest of the specifications.

Figure 2 presents the Root-Mean-Square (RMS) error in the regression as a function of the number of MC parameters selected by the search algorithm, for the complete set of training instances (marked with blue diamonds), and the samples in the independent verification set (marked with red circles). For illustration purposes, instead of setting a target prediction error as stopping condition for the algorithm, the search has been set to find and rank the 20 most relevant MC parameters, as shown in Fig. 2. It is interesting to notice that the proposed algorithm guides the selection of MC parameters in such a way that it yields a monotonic improvement of the prediction error. Moreover, it is clear to see that this improvement of the prediction error tends to slow down around the fifteenth iteration of the search. In this line, the 15 most significant parameters have been selected and are labelled to the corresponding data points in the figure. Figure 3 shows the scatterplot of the predicted versus actual gain of the PA when



Fig. 2. Exploration of the MC process parameter space for finding the root causes of gain parametric variation for the DUT. Data point labels indicate the MC process parameter $M C_{i}$ selected in each iteration of the search algorithm.

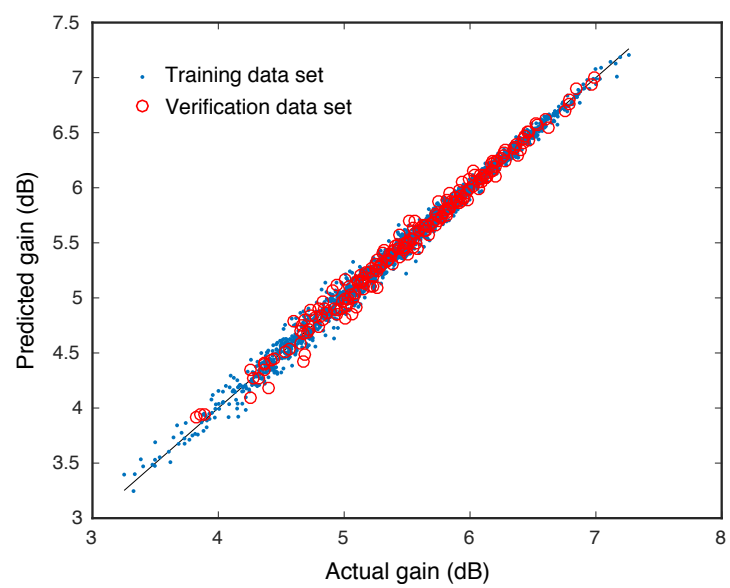

Fig. 3. Scatterplot of predicted versus actual gain of the PA using the 15 most relevant MC parameters as signatures.

we train a Neural Network regression model using these 15 selected MC parameters as input features. Blue dots represent the training data set, while red circles mark the samples in the independent verification set. It is clear that the selected MC parameters have a very strong correlation to the target performance.

Once the most relevant MC parameters for the prediction of the targeted performance have been identified, the second step in the presented test design methodology is to propose 
appropriate non-intrusive sensors that yield signatures strongly correlated to these parameters. The design of these sensors and their associated measurements is guided by the physical meaning of the selected MC parameters. Although it has to be noted that for confidentiality reasons we cannot disclose the actual Monte Carlo models in the PDK of the technology, for illustration purposes we can detail that parameters $M C_{46}$, $M C_{48}, M C_{49}, M C_{60}, M C_{69}, M C_{150}$ and $M C_{154}$ are linked to the electrical properties of transmission lines, parameters $M C_{12}$, and $M C_{23}$ point to $\mathrm{MOM}$ capacitor variations, and the rest of identified MC parameters $\left(M C_{77}, M C_{78}, M C_{80}\right.$, $M C_{85}, M C_{86}$, and $\left.M C_{111}\right)$ are related to variations of the NMOS transistor.

Taking into account the physical meaning of the parameters, Table II details the schematics of the developed sensors and the associated measurements (i.e. signatures). In order to verify that the 15 selected MC parameters are covered by the proposed signatures, we perform our Brownian distance correlation-directed search in the space of process parameters for regressing each one of the proposed signatures. In this line, Table II also includes the list of most significant MC parameters that explain the variation of each proposed signature for validation. As it can be seen, all 15 relevant MC parameters are covered by the devised signatures.

In total, for covering the identified 15 most significant MC parameters we have developed 5 non-intrusive sensors and 9 signatures labelled as $\left\{S_{1}, \ldots, S_{9}\right\}$. Notice that signatures are sensitive to multiple MC parameters, which reduces the number of necessary measurements. The proposed set of signatures contains DC measurements and low frequency AC measurements extracted at $100 \mathrm{MHz}$. These low frequency measurements are simpler and cheaper to extract than at-speed $60 \mathrm{GHz}$ measurements on the PA under test. From an electrical point of view, the proposed signatures contain information about phase-shifts in the elements of the PA $\left(S_{1}, S_{2}, S_{4}\right)$, capacitive behavior $\left(S_{6}\right.$, and $\left.S_{7}\right)$, electrical losses $\left(S_{3}, S_{5}\right.$, and $\left.S_{8}\right)$ and the operation point of the NMOS transistor $\left(S_{9}\right)$.

The developed non-intrusive sensors and their corresponding signatures are then stored in a library for reuse in future indirect test programs. As it was mentioned above, the developed sensors are independent of the DUT topology, since they are devised to target specific root causes of parametric variation in a given technology. The reuse of the library of nonintrusive sensors for other DUTs offers an additional layer of automation to the proposed test design strategy.

As a final validation of the performance of the proposed assisted test design strategy, we applied the resulting nonintrusive machine learning indirect test to the PA. A perceptron Neural Network is trained to regress the gain of the PA under test from the identified 9 input signatures $\left\{S_{1}, \ldots, S_{9}\right\}$ extracted from the generated non-intrusive sensors. Figure 4 shows the scatterplot of the predicted versus actual gain over the 2000 generated instances of the PA under test. Blue dots mark the training data set, while red circles represent the samples in the independent verification set. As it can be seen, the proposed test program offers a good estimation of the



Fig. 4. Scatterplot of predicted versus actual gain of the PA using the generated indirect test program with 9 non-intrusive signatures.

gain performance across the complete variation range. The RMS error of the prediction for samples in the independent verification set is of only $0.08 \mathrm{~dB}$, which is comparable to the regression in Fig. 3 that employed the 15 most significant MC process parameters as input signatures and yields an RMS prediction error of $0.07 \mathrm{~dB}$. This result is a good indicator of the relevance of the proposed set of signatures to capture the variation of the target performance.

Finally, the complete test design process can be repeated for the rest of the specifications of the PA under test. Interestingly, if we consider the complete set of PA performances in Table I, the described assisted test design algorithm leads to an additional measurement on one of the already proposed nonintrusive sensors. This new signature, $S_{10}$ using the previous notation, is the imaginary part of scattering parameter $S_{11}$ measured at $100 \mathrm{MHz}$ for the MOS transistor sensor described in Table II. In order to validate the resulting non-intrusive indirect tests, perceptron Neural Network models have been trained for regressing the complete set of PA specifications using the identified set of signatures $\left\{S_{1}, \ldots, S_{10}\right\}$. Table III lists the RMS prediction error for samples in the independent verification set for each specification. In order to evaluate the quality of the regression models, Table III compares the obtained RMS errors with the standard variation of the specification in MC simulations. It is clear to see that the obtained models trained with the automatically identified set of signatures offer significant predictions for all the considered specifications.

\section{CONCLUSIONS}

We have presented a systematic methodology for generating a non-intrusive indirect test program for mm-wave circuits. The proposed strategy represents a promising step towards the fully automation of test generation for non-intrusive machine learning test. The proposed methodology is illustrated using a $60 \mathrm{GHz}$ PA case study designed in STMicroelectronics 55 nm BiCMOS technology. We have shown that the presented assisted test design algorithm enables us to find the root causes of parametric performance degradation and guides the design of appropriate non-intrusive sensors and signatures for accurate performance prediction. 
TABLE II

DEVELOPED NON-INTRUSIVE SENSORS AND ASSOCIATED SIGNATURES FOR GAIN PREDICTION

\begin{tabular}{|c|c|c|}
\hline Process variation sensor schematic & Signature description & MC parameters covered \\
\hline Input & $\begin{array}{l}\text { - } S_{1} \text { : Imaginary part of } S_{11} @ 100 \mathrm{MHz} \\
\text { - } S_{2}: \text { Imaginary part of } S_{21} @ 100 \mathrm{MHz} \\
\text { - } S_{3}: \text { Resistance }\end{array}$ & $\begin{array}{l}-M C_{46}, M C_{48}, M C_{49}, M C_{69}, M C_{150} \\
-M C_{46}, M C_{48}, M C_{69}, M C_{150}, M C_{154} \\
\text { - } M C_{48}\end{array}$ \\
\hline Input $\underset{\substack{\mathrm{L}=500 \mu \mathrm{m} \\
\mathrm{W}=18.6 \mu \mathrm{m}}}{\mathrm{C}}$ Wide microstrip line & - $S_{4}$ : Imaginary part of $S_{11} @ 100 \mathrm{MHz}$ & - $M C_{69}, M C_{150}, M C_{154}, M C_{60}, M C_{46}$ \\
\hline Input & $\begin{array}{l}\text { - } S_{5} \text { : Real part of } Y_{11} @ 100 \mathrm{MHz} \\
\text { - } S_{6}: \text { Imaginary part of } S_{21} @ 100 \mathrm{MHz}\end{array}$ & $\begin{array}{l}\text { - } M C_{12}, M C_{31} \\
\text { - } M C_{23}, M C_{31}\end{array}$ \\
\hline Input & - $S_{7}$ : Imaginary part of $S_{11} @ 100 \mathrm{MHz}$ & - $M C_{86}, M C_{85}, M C_{111}, M C_{91}$ \\
\hline $\begin{array}{l}\text { NMOS transistor } \\
1 \mathrm{~V} \quad \underline{1.2 \mathrm{~V}} \\
\end{array}$ & & \\
\hline$\underset{\mathrm{W}=72 \mu \mathrm{m}}{\mathrm{L}=0.06 \mu \mathrm{m}}$ & $\begin{array}{l}\text { - } S_{8}: \text { Gate resistance } \\
\text { - } S_{9}: \text { Bias current }\end{array}$ & $\begin{array}{l}\text { - } M C_{80}, M C_{111}, M C_{4}, M C_{77}, M C_{78} \\
\text { - } M C_{11}, M C_{91}, M C_{35}\end{array}$ \\
\hline
\end{tabular}

TABLE III

PREDICTION OF PA PERFORMANCES USING THE GENERATED INDIRECT TEST PROGRAM

\begin{tabular}{llcc}
\hline Specification & Unit & $\sigma_{\text {spec }}$ & RMS prediction error \\
\hline Gain $\left(S_{21}\right)$ & $\mathrm{dB}$ & 0.69 & 0.08 \\
PAE & $\%$ & 1.98 & 0.17 \\
$S_{11}$ & $\mathrm{~dB}$ & 4.46 & 0.5 \\
$S_{22}$ & $\mathrm{~dB}$ & 4.92 & 0.7 \\
$S_{12}$ & $\mathrm{~dB}$ & 0.75 & 0.13 \\
$C_{1 d B}$ & $\mathrm{dBm}$ & 0.58 & 0.075 \\
$P_{\text {sat }}$ & $\mathrm{dBm}$ & 0.17 & 0.025 \\
$I I P 3$ & $\mathrm{dBm}$ & 0.67 & 0.11 \\
\hline
\end{tabular}

\section{REFERENCES}

[1] A. Hajimiri et al., "Integrated Phased Array Systems in Silicon," Proceedings of the IEEE, vol. 93, no. 9, pp. 1637-1655, Sept 2005.

[2] W. Roh et al., "Millimeter-wave beamforming as an enabling technology for 5G cellular communications: theoretical feasibility and prototype results," IEEE Communications Magazine, vol. 52, no. 2, pp. 106-113, February 2014.

[3] J. Bae, Y. S. Choi, J. S. Kim, and M. Y. Chung, "Architecture and performance evaluation of MmWave based 5G mobile communication system," in 2014 International Conference on Information and Communication Technology Convergence (ICTC), Oct 2014, pp. 847-851.

[4] P. N. Variyam and A. Chatterjee, "Enhancing test effectiveness for ana$\log$ circuits using synthesized measurements," in VLSI Test Symposium, 1998, pp. 132-137.

[5] A. Zjajo, M. J. Barragan, and J. P. de Gyvez, "BIST Method for DieLevel Process Parameter Variation Monitoring in Analog/Mixed-Signal Integrated Circuits," in 2007 Design, Automation \& Test in Europe Conference \& Exhibition, 2007, pp. 1-6.

[6] L. Abdallah, H. G. Stratigopoulos, and S. Mir, "True non-intrusive sensors for RF built-in test," in 2013 IEEE International Test Conference (ITC), 2013, pp. 1-10.

[7] A. Dimakos et al., "Built-in test of millimeter-wave circuits based on non-intrusive sensors," in 2016 Design, Automation \& Test in Europe Conference \& Exhibition (DATE), 2016, pp. 505-510.

[8] P. N. Variyam and A. Chatterjee, "Specification-driven test generation for analog circuits," IEEE Transactions on Computer-Aided Design of Integrated Circuits and Systems, vol. 19, no. 10, pp. 1189-1201, 2000.

[9] M. Barragan and G. Leger, "A Procedure for Alternate Test Feature Design and Selection," IEEE Design \& Test, vol. 32, no. 1, pp. 18-25, Feb. 2015.

[10] H.-G. Stratigopoulos, P. Drineas, M. Slamani, and Y. Makris, "RF Specification Test Compaction Using Learning Machines," Trans. on VLSI Systems, 2009.

[11] S. Larguech et al., "Efficiency evaluation of analog/RF alternate test: Comparative study of indirect measurement selection strategies," $M i$ croelectronics Journal, vol. 46, no. 11, pp. 1091-1102, Nov. 2015.

[12] A. Gomez-Pau, L. Balado, and J. Figueras, "Efficient Production Binning Using Octree Tessellation in the Alternate Measurements Space," Trans. on Computer-Aided Design of Int. Circ. and Syst., vol. 35, no. 8, pp. 1386-1395, 2016

[13] H. Ayari et al., "Smart selection of indirect parameters for DC-based alternate RF IC testing," in VLSI Test Symposium, 2012, pp. 19-24.

[14] M. J. Barragan and G. Leger, "Efficient selection of signatures for analog/RF alternate test," in European Test Symposium, 2013.

[15] G. Leger and M. J. Barragan, "Brownian distance correlation-directed search: A fast feature selection technique for alternate test," Integration, the VLSI Journal, vol. 55, pp. 401-414, Sep. 2016.

[16] L. Abdallah, H. G. Stratigopoulos, S. Mir, and C. Kelma, "Experiences with non-intrusive sensors for RF built-in test," in 2012 IEEE International Test Conference, 2012, pp. 1-8.

[17] G. J. Székely and M. L. Rizzo, "Brownian distance covariance," The Annals of Applied Statistics, vol. 3, no. 4, pp. 1236-1265, 2009. 